\title{
One Health and Hendra virus: a collaborative approach in action
}

\author{
Belinda Crawford $^{\mathrm{A}}$, Ian Roth ${ }^{\mathrm{B}}$ and Tiggy Grillo ${ }^{\mathrm{C}}$ \\ ${ }^{\mathrm{A}}$ NSW Public Health Officer Training Program, NSW Ministry \\ of Health \\ ${ }^{\mathrm{B}}$ Department of Primary Industries \\ ${ }^{\mathrm{C}}$ Australian Wildlife Health Network
}

The One Health initiative (http://www.onehealthinitiative. com/) encompasses the health of humans, animals and the environment, recognising the indivisible interconnections that exist between these domains. As over $60 \%$ of emerging and re-emerging diseases are transmitted from animals to humans (zoonoses), the One Health initiative has significant potential to reduce the global health threat caused by infectious diseases. ${ }^{1}$

The concept of One Health is not new as the union of human and animal health has long been understood and accepted; however our view of zoonoses has traditionally been compartmentalised within public health and veterinary medicine, with little attention given to environmental health elements and cross-disciplinary collaborations. The One Health approach has gained increasing global attention, marked by the partnerships of major human health, animal health and environmental organisations. An integrated One Health approach requires commitment at global, national, state and local levels. ${ }^{2}$

The management of zoonoses in New South Wales (NSW) involves human, animal and environmental health sectors. For human health, the Centre for Health Protection, NSW Ministry of Health, is responsible for policy development and the coordination of statewide surveillance and response activities. NSW public health units detect and respond to local infectious disease issues and work collaboratively with members of the One Health team. For animal health, the Department of Primary Industries (DPI) oversees the health of livestock and companion animals. Regional veterinary officers work closely with local stakeholders, including human and environmental health experts. The NSW Office of Environment and Heritage monitors the health of free-ranging wildlife and zoo collection animals. Wildlife disease events are reported to the Australian Wildlife Health Network, who maintain the national wildlife disease surveillance database. ${ }^{3}$

\section{Hendra virus infection: a One Health approach}

Hendra virus infection was first described after an outbreak of severe respiratory disease in 18 horses and two humans in Brisbane in 1994. To date, there have been 24 cases in
Queensland and nine in northern NSW. Horses appear to be incidental hosts following exposure to Flying-foxes or their excretions. Horse-to-horse transmission occurs infrequently and is more likely when horses are in very close contact. Infected horses usually experience rapid deterioration from brief but severe respiratory or neurological disease with a high case fatality rate. One dog became serologically positive probably after contact with an infected horse. In seven cases, Hendra virus infection has spread from horses to humans following close contact and has resulted in pneumonic or encephalitic illnesses. Four of these people have died. There is no evidence of human infection from other sources, such as direct contact with Flying-foxes. There is no known treatment and clinical management has been based on supportive measures. ${ }^{4}$

Hendra virus in horses is a notifiable disease in all Australian jurisdictions. If suspected, the NSW DPI works with NSW Health to complete a prompt epidemiological investigation to identify and monitor people at risk and provide advice to minimise the risk of infection to humans and other horses. The property where the horse cases are located is quarantined and animals that are infected are euthanased following guidelines in the AUSVETPLAN Response Policy Briefs. The human health response follows the Hendra Virus National Guideline for Public Health Units and includes the provision of information for all people at risk and testing and follow-up on a caseby-case basis. Open and transparent communication between all team members and to the general public is an important component of Hendra virus investigations. ${ }^{4}$ The formation of the cross-border Intergovernmental Hendra Virus Taskforce further demonstrates support for the One Health approach in minimising the impacts of this potentially devastating disease on human and animal health.

\section{References}

1. Jones KE, Patel NG, Levy MA, Storeygard A, Balk D, Gittleman JL et al. Global trends in emerging infectious diseases. Nature 2008; 451: 990-3. doi:10.1038/nature06536

2. Atlas R, Rubin C, Maloy S, Daszak P, Colwell R, Hyde B. One Health - attaining optimal health for people, animals, and the environment. Microbe 2010; 5(9): 383-9.

3. Adamson S, Marich A, Roth I. One Health in NSW: coordination of human and animal health sector management of zoonoses of public health significance. N S W Public Health Bull 2011; 22(5-6): 105-12. doi:10.1071/NB11003

4. Hess IMR, Massey PD, Walker B, Middleton DJ, Wright TM. Hendra virus: what do we know? NS W Public Health Bull 2011; 22(5-6): 118-22. doi:10.1071/NB10077 